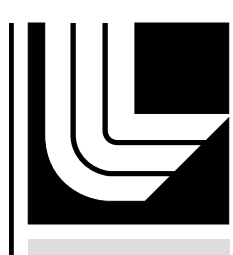

LAW RENCE LIVERMORE N A T IO N A L LABORATORY

\title{
High Power Picosecond Laser Pulse Recirculation
}

M. Y. Shverdin, I. Jovanovic, V. A. Semenov, S. M. Betts, C. Brown, D. J. Gibson, R. M. Shuttlesworth, F. V. Hartemann, C. W. Siders, C. P. Barty

April 15, 2010

Optics Letters 
This document was prepared as an account of work sponsored by an agency of the United States government. Neither the United States government nor Lawrence Livermore National Security, LLC, nor any of their employees makes any warranty, expressed or implied, or assumes any legal liability or responsibility for the accuracy, completeness, or usefulness of any information, apparatus, product, or process disclosed, or represents that its use would not infringe privately owned rights. Reference herein to any specific commercial product, process, or service by trade name, trademark, manufacturer, or otherwise does not necessarily constitute or imply its endorsement, recommendation, or favoring by the United States government or Lawrence Livermore National Security, LLC. The views and opinions of authors expressed herein do not necessarily state or reflect those of the United States government or Lawrence Livermore National Security, LLC, and shall not be used for advertising or product endorsement purposes. 


\title{
High Power Picosecond Laser Pulse Recirculation
}

\author{
M. Y. Shverdin, I. Jovanovic, V. A. Semenov, S. M. Betts, C. Brown, D. J. Gibson, \\ R. M. Shuttlesworth, F. V. Hartemann, C. W. Siders, C. P. J. Barty \\ Lawrence Livermore National Laboratory, L-460, P. O. Box 808, Livermore, California 94550, USA \\ ${ }^{*}$ Corresponding author: shverdin2@llnl.gov
}

Compiled April 12, 2010

We demonstrate a nonlinear crystal-based short pulse recirculation cavity for trapping the second harmonic of an incident high power laser pulse. This scheme aims to increase the efficiency and flux of Compton-scattering based light sources. We demonstrate up to $36 \mathrm{x}$ average power enhancement of frequency doubled sub-millijoule picosecond pulses, and $17 \mathrm{x}$ average power enhancement of $177 \mathrm{~mJ}, 10 \mathrm{ps}, 10 \mathrm{~Hz}$ pulses. (C) 2010 Optical Society of America

OCIS codes: (190.7110); (190.4360) Nonlinear optics, four-wave mixing

In many applications of high intensity lasers such as Thomson scattering, cavity ring down spectroscopy and high-harmonic generation in short gas jets, the incident laser beam is negligibly modified by the interaction. In Compton scattering light sources, for example, the conversion efficiency from laser photons to x-rays is typically below $10^{-9}$. Reusing the residual laser pulse after each interaction increases the generation efficiency of the process. In this letter, we present a novel, high energy pulse recirculation scheme based on injection and trapping a single laser pulse inside a passive optical cavity. A thin nonlinear crystal acts as an optical switch, trapping the frequency converted light. This technique, termed recirculation injection by nonlinear gating (RING) is compatible with joule class, 100s of Watts of average power, picosecond laser pulses.

Existing pulse recirculation schemes rely on either resonant cavity coupling $[1,2]$ or active (electro-optic or acousto-optic) pulse switching [3,4] into and out of the resonator. Active pulse switching schemes are suitable for low intensity, nanosecond duration pulses [5]. Resonant cavity coupling requires interferometric cavity alignment and $\mathrm{MHz}$ repetition rates. To date, researchers have attained up to $100 \mathrm{x}$ enhancement for $1 \mathrm{~W}$ average power, $\approx 50$ fs duration incident pulses with per pulse energy $<1 \mu \mathrm{J}$. We have previously described the concept of RING along with preliminary results [6].

The primary motivation for RING is the average brightness enhancement of high peak power $\gamma$-rays generated by laser Compton scattering. A new generation of monoenergetic $\gamma$-ray sources utilize joule-class, $\approx 10$ ps interaction laser focused to overlap with a counterpropagating, relativistic, low emittance, electron beam. The principle of RING is illustrated in Fig. 1(b). The incident $(1 \omega)$ laser pulse is injected into the cavity through a highly transmissive dichroic mirror and is then frequency doubled. The nonlinear crystal acts as an optical switch, modifying both the frequency and the polarization of the incident light. The crystal thickness is optimized for high conversion efficiency. The dichroic resonator mirrors transmit at $1 \omega$ and reflect at $2 \omega$, trapping the up-

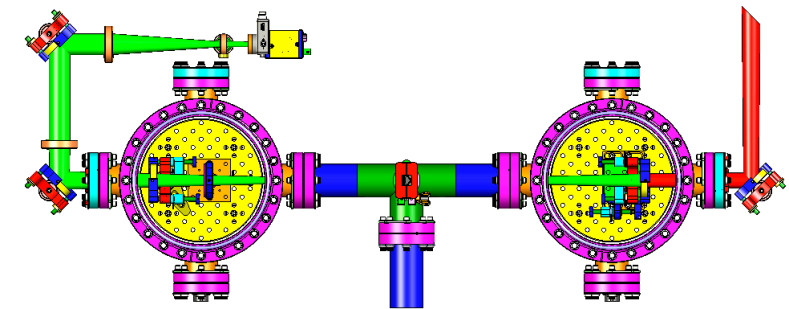

(a)

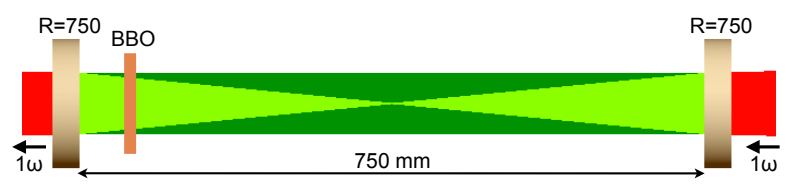

(b)

Fig. 1. (a) RING cavity design showing two vacuum chambers that contain the nonlinear crystal and dichroic mirrors. (b) Raytraced beam inside and outside the RING cavity.

converted $2 \omega$ pulse inside the cavity. An interaction of the laser pulse with e. g. an electron beam would occur at the internal cavity focus. After repeated roundtrips inside the cavity, the laser pulse decays due to Fresnel losses at the crystal faces and cavity mirrors, and hard edge diffraction losses. We implement RING cavity in a Fabry-Perot configuration (Fig. 1(b)) to maximize the cavity finesse. The confocal arrangement relay images any plane within the cavity back onto itself, which minimizes diffraction losses and supports an arbitrary incident spatial profile. Compared to active pulse switching, the main advantage of RING is an order of magnitude reduction of the accumulated nonlinear phase with each roundtrip. For typical incident pulse intensities of $1-10 \mathrm{GW} / \mathrm{cm}^{2}$, the nonlinear crystal thickness is a few $\mathrm{mm}$, compared to a few cm thick crystal inside a Pockels cell.

Here, we report on both millijoule and joule scale pulse 
recirculation. The difference in the RING cavity designs for the two experiments involves the aperture size of the optical components and the choice of the doubling crystal. The experimental design is shown in Fig. 1(a) (b). Two concave $750 \mathrm{~mm}$ radius of curvature (ROC) mirrors spaced approximately $750 \mathrm{~mm}$ apart form a confocal resonator. The mirrors' multi-layer dielectric coating on the surface internal to the cavity is $99.8 \%$ reflective at $527 \mathrm{~nm}$ and $98 \%$ transmissive at $1053 \mathrm{~nm}$. The mirrors' flat surface external to the cavity is anti-reflection coated at both wavelengths.

In the low energy experiment, we inject a $10 \mathrm{~Hz}$, $1.7 \mathrm{~mJ}, 10 \mathrm{~nm}$ bandwidth pulse centered at $1053 \mathrm{~nm}$ and chirped to $2.25 \mathrm{ps}$ through the right 1" diameter cavity mirror, which is a negative lens in transmission. Upstream beam sizing optics produce a collimated $\mathrm{w}_{0}=4 \mathrm{~mm}$ gaussian inside the cavity. A $10 \mathrm{x} 10 \mathrm{x} 1 \mathrm{~mm}$ Type I SHG BBO crystal is next to the end cavity mirror. The crystal has a single layer $\mathrm{MgF}_{2} \mathrm{AR}$ coating at both wavelengths $(0.3 \%$ loss per surface). The choice of $\mathrm{BBO}$ is motivated by its excellent thermomechanical properties, broad spectral and thermal acceptance, and a relatively low nonlinear index to effective nonlinear coefficient ratio, $\mathrm{n}_{2} / \mathrm{d}_{\text {eff. }}$. We measure $270 \mu \mathrm{J}$ at $527 \mathrm{~nm}$ after the crystal, corresponding to peak intensity $\approx 0.8 \mathrm{GW} / \mathrm{cm}^{2}$. The majority of the residual $\mathrm{IR}$ is coupled out of the cavity through the end mirror. We observe ghost reflections from crystal and mirror surfaces which complicate cavity alignment. The cavity length, $L_{c a v}=\mathrm{ROC}+\Delta L$, where $\Delta L=L_{c}\left(1-1 / n_{c}\right)$. Here, $L_{c}$ is the crystal's thickness and $n_{c}$ is its refractive index at $2 \omega . \Delta L=2 \mathrm{~mm}$ for our parameters. Inside the cavity, the $2 \omega$ beam is collimated when traveling from right to left, and focuses in the middle when traveling in the opposite direction. The cavity is contained inside two interconnected vacuum chambers. Remotely controlled actuators adjust the tip and tilt of both cavity mirrors, the phasematching angle of the nonlinear crystal, and the cavity length. The chambers are pumped down to $10^{-3}$ Torr range to minimize nonlinear phase accumulation and prevent air breakdown.

We measure cavity enhancement at $2 \omega$, defined as enh $=\sum_{n=0}^{N} I_{n} / I_{0}$, where $I_{n}$ is pulse power after $\mathrm{n}$ roundtrips, and $N$ is the total number of roundtrips, by analyzing the cavity ring-down signal. The leakage $527 \mathrm{~nm}$ light passes through the end mirror, and is collected on a $1.2 \mathrm{GHz}$ Si photodiode (EOT-2030) and sampled by a $15 \mathrm{GHz}$ oscilloscope. Dichroic mirrors and green bandpass filters scrape off any residual IR from the detected beam. Fig. 2(a) shows the measured signal consisting of approximately 170 pulses spaced by the $5 \mathrm{~ns}$, cavity roundtrip time. Impedance mismatch between the photodiode and the oscilloscope introduces some ringing in the recorded waveform.

The circles in Fig. 2(a) correspond to the normalized power in each pulse, determined by integrating the voltage signal. The expected power in each successive pulse is $(1-\alpha)$ times the power of the previous pulse,

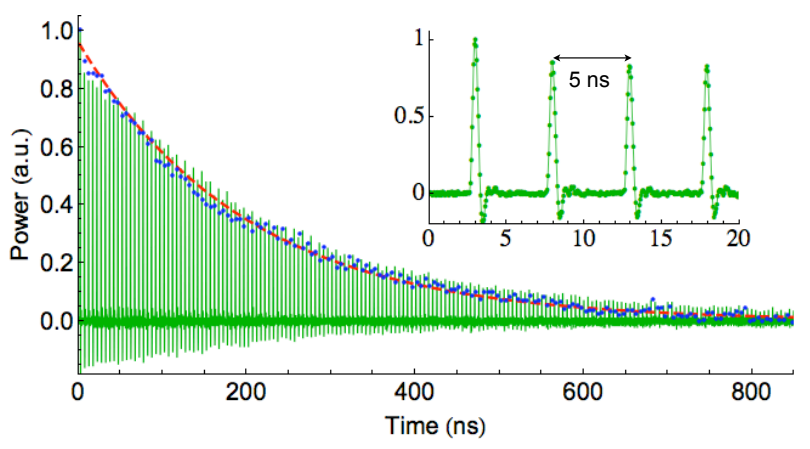

(a)

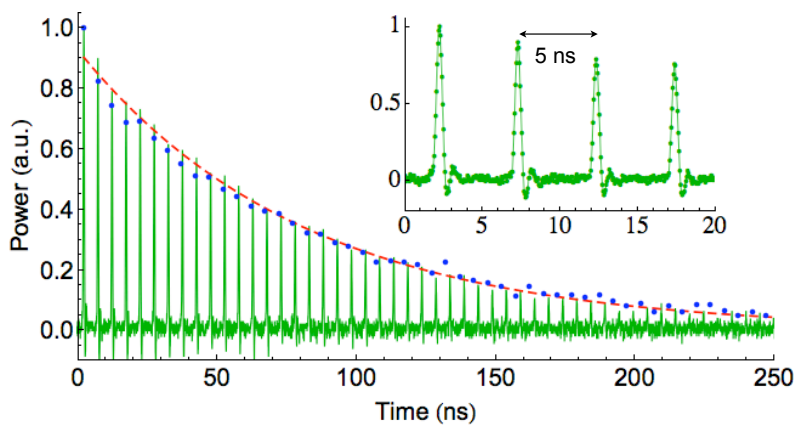

(b)

Fig. 2. Cavity ring-down signal for (a) $270 \mu \mathrm{J}$ and (b) $177 \mathrm{~mJ} 2 \omega$ beam. A dashed line is a fit to the residual power (small circles) in the pulse after each roundtrip. The figure inset shows the 5 ns pulse to pulse spacing.

or $I_{n}=I_{0}(1-\alpha)^{n}$, where $I_{0}$ is the initial signal power and $\alpha$ is the loss per roundtrip. The total cavity enhancement is then $\left[1-(1-\alpha)^{n}\right] / \alpha$. The dashed line in the figure is a fit to pulse power with $\alpha=0.025$, corresponding to $e n h=40$. Contributions above the $1.8 \%$ Fresnel losses, could be attributed to hard edge diffraction and scattering in the nonlinear crystal. In the measured trace, the ratio of the powers in the first to the second pulses is larger than for subsequent pulses; an effect caused by additional diffraction losses during the first pass. The waveform also exhibits "picket fence" effect, where the power of many pulses is higher than of the adjacent pulses. This is likely caused by a slight cavity misalignment. We explicitly calculate cavity enhancement by summing over all of the observed pulses, obtaining $e n h=36$. We estimate the total accumulated nonlinear phase, $\phi_{N L}=\frac{2 \pi n_{2} L_{c} I_{\text {peak }}}{\lambda} \frac{1-(1-\alpha)^{N}}{\alpha}=0.7 \mathrm{rad}$, where, $n_{2}$ is the nonlinear refractive index $\left(8.8 \times 10^{-16} \mathrm{~cm}^{2} / \mathrm{W}\right)$.

In the high energy experiment, we utilized a hyperdispersion CPA system under development at LLNL [7]. Both the spatial and the temporal profiles exhibited significant aberrations. We injected $10 \mathrm{~Hz}, 677 \mathrm{~mJ}, 0.25 \mathrm{~nm}$ bandwidth pulses at $1064 \mathrm{~nm}$ with a FWHM pulse duration of $20 \mathrm{ps}$. Autocorrelation measurements of the IR pulse indicate that $70 \%$ of the $677 \mathrm{~mJ}$ is contained in a wide 400 ps pulse pedestal. Compared to the low energy experiment, the main changes to the RING cavity 
involve replacing 1" diameter cavity mirrors with 2" diameter mirrors and replacing the small aperture BBO crystal with 30x30x6 mm deuterated potassium dihydrogen phosphate (DKDP) cut for Type II phasematching. After frequency doubling, we generate $177 \mathrm{~mJ}$ at $532 \mathrm{~nm}$ in an slightly elliptical $12 \times 15 \mathrm{~mm}$ FWHM beam. Computer simulations indicate that the pulse at $532 \mathrm{~nm}$ is 16 ps FWHM and $50 \%$ of total energy is contained in the wide pedestal. We estimate the resulting peak pulse intensity at $4 \mathrm{GW} / \mathrm{cm}^{2}$.

The high energy ring-down signal is shown in Fig. 2(b). We observe pulses over $\approx 50$ roundtrips. Here, the loss coefficient, $\alpha=0.06$, resulting in cavity enhancement, $e n h=17$, the same value as obtained by explicitly summing the powers in each pulse. The estimated total nonlinear phase is $2.8 \mathrm{rad}$. We attribute the significant degradation in cavity enhancement to the poor spatial beam quality of the high energy $1 \omega$ laser. The near field spatial profile suffers from high frequency intensity modulation causing high hard edge diffraction losses at the crystal. When we replaced the DKDP crystal with a smaller aperture 20x20x1.2 mm BBO crystal, higher diffraction losses reduced cavity enhancement to 11 .

RING cavity design is scalable to very high peak and average power recirculation. Linear absorption in the crystal is the primary limitation to the maximum sustainable average power inside the cavity. LBO, YCOB, and $\mathrm{BBO}$ crystals are particularly attractive candidates for high average power operation. YCOB and LBO are also available in a large aperture, allowing scaling to high peak powers. For a simple edge cooling scheme, finite element simulation of the thermal profile inside the crystal indicate that LBO and YCOB support up to $1.2 \mathrm{~kW}$ of total recirculating power. Assuming cavity enhancement of 30 and $50 \%$ conversion efficiency, this corresponds to $75 \mathrm{~W}$ in the incident $1 \omega$ pulse. The peak recirculating power limit could be significantly increased with surface cooling schemes.

Nonlinear phase accumulation in the crystal is the primary limit to peak power scaling. For a gaussian pulse, the bandwidth doubles for $\phi_{N L} \approx 2.4$. In monoenergetic gamma-ray generation, this increases the bandwidth of the generated photons [8]. Other deleterious effects include whole beam self-focusing and modulation instability growth. Increasing beam size and correspondingly the aperture of the cavity optics mitigates nonlinear phase accumulation. DKDP crystals are available in sizes up to $40 \mathrm{x} 40 \mathrm{~cm}$, potentially enabling recirculation of $100 \mathrm{~J}, 10 \mathrm{ps}$ pulses.

Highest attainable cavity enhancement is a function of roundtrip cavity losses and $\phi_{N L}$. Assuming that $\phi_{N L} \leq 2.5$, we calculate cavity enhancement vs loss per roundtrip (Fig 3). Cavity enhancement is plotted for three different pulse intensities. We assume LBO parameters for the nonlinear medium, with $\mathrm{n}_{2}=3 \times 10^{-16} \mathrm{~cm}^{2} / \mathrm{W}$ and $L_{c}=2 \sqrt{I / 10}$. Crystal thickness, $L_{c}$ and initial $2 \omega$ pulse intensity, $I$, are specified in $\mathrm{mm}$ and $\mathrm{GW} / \mathrm{cm}^{2}$, respectively. We also assume a cavity

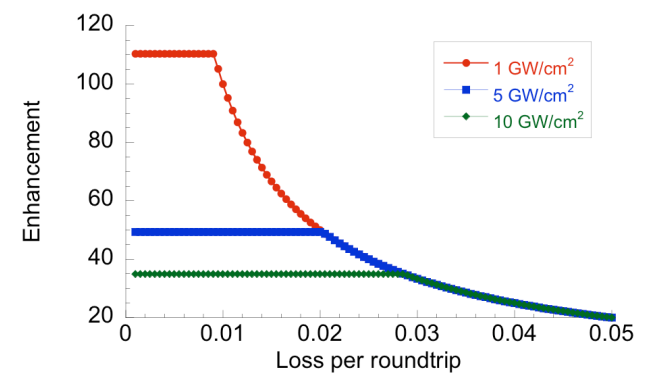

Fig. 3. Simulation of maximum cavity enhancement vs the loss per cavity roundtrip for three incident pulse intensities. We assume that LBO is the nonlinear switch and $\phi_{N L}<2.5$.

configuration where the nonlinear crystal is single-passed in each roundtrip. From the plot, when $I=5 \mathrm{GW} / \mathrm{cm}^{2}$, the maximum enhancement of 50 is achieved for $\alpha<0.02$ given sufficient number of roundtrips. As cavity loss decreases below $0.02, \phi_{N L}=2.5$ limit is reached in fewer roundtrips. For $\alpha>0.02, \phi_{N L}<2.5$.

We presented a novel pulse recirculation design suitable for trapping short, high peak power pulses. RING recirculation minimizes pulse dispersion and nonlinear phase accumulation which limits the performance of active pulse switching schemes. We achieved 36x cavity enhancement for sub $\mathrm{mJ}$ and $17 \mathrm{x}$ enhancement for $177 \mathrm{~mJ}$, few picosecond pulses. Deploying RING on a Compton scattering light source could lead to more than an order of magnitude increase in average source brightness of the generated $\gamma$-ray flux.

This work was performed under the auspices of the U.S. Department of Energy by University of California, Lawrence Livermore National Laboratory under Contract W-7405-ENG- 48. We also acknowledge support of DOE/NA-22.

Prepared by LLNL under Contract DE-AC52-07NA27344.

\section{References}

1. C. Gohle, T. Udem, M. Herrmann, J. Rauschenberger, R. Holzwarth, H. Schuessler, F. Krausz, and T. Hänsch, Nature 436, 234 (2005).

2. R. J. Jones, K. D. Moll, M. J. Thorpe, and J. Ye, Phys. Rev. Lett. 94, 193201 (2005).

3. D. Yu and B. Stuart, Opt. Commun. 214, 291 (2002).

4. T. Mohamed, G. Andler, and R. Schuch, Opt. Commun. 214, 291 (2002).

5. D. Meng, F. Sakamoto, T. Yamamoto, K. Dobashi, M. Uesaka, H. Nose, D. Ishida, N. Kaneko, and Y. Sakai, Nucl. Instrum. Methods Phys. Res. B 261, 52 (2007).

6. I. Jovanovic, M. Shverdin, D. Gibson, and C. Brown, Nucl. Instrum. Methods Phys. Res. B 578, 160 (2007).

7. D. Gibson, to be published 00, 00 (2010).

8. F. Albert, S. Anderson, G. Anderson, S. Betts, D. Gibson, C. Hagmann, M. Johnson, M. Messerly, V. Semenov, M. Shverdin, A. Tremaine, F. Hartemann, C. Siders, D. McNabb, and C. Barty, Opt. Lett. 0, 0 (2010). 